\title{
Primary arthrodesis in acute ligamentous lisfranc injuries
}

A.A.Salama, M.S.Abouzied and M.O.Hegazy

Orthopedic, Dept., Faculty of Medicine, Benha Univ., Benha, Egypt

Email: Ahmed Salama@gmail.com

\begin{abstract}
Lisfranc wounds influence the tarsometatarsal (TMT), intercuneiform, and the naviculocuneiform joints. It very well may be bony, ligamentous, or a mix of the two. The meaningful step forward somewhat recently has been the accentuation on early stable anatomical decrease and adjustment of these wounds. Late examinations have recommended that essential arthrodesis might be a favored method for basically ligamentous Lisfranc wounds. This investigation expected to assess the momentary consequences of essential arthrodesis in unadulterated ligamentous lisfranc wounds. Twenty patients, 13 guys and 7 females with a mean time of $27.4 \pm 6.19$ with least age 19 years of age and greatest age 39 years of age were remembered for this investigation. The most widely recognized instrument of injury was street auto collision $(55 \%)$, trailed by tumble from stature $(40 \%)$, and followed by hyper plantar flexion foot injury during plunging steps (5\%). the mean AOFAS score of the included patients was $81.65 \pm 1.60$ with least score 80 and greatest score 84 . the mean EFAS score of the included patients was $31.60 \pm 1.76$ with least score 28 and most extreme score 34 . the mean Pain VAS score of the included patients was $2.05 \pm 0.76$ with least score 1 and greatest score 3 . the mean Union season of the included patients was $12.55 \pm 0.51$ weeks with least 12 weeks and most extreme 13 weeks. All in all, Lisfranc wounds are unpredictable and care should be taken in choosing the fitting treatment. Essential arthrodesis in unadulterated ligamentous lisfranc injury has benefits: diminished foot deformation rates, supported biomechanical morphology of the feet, diminished intricacies, more elevated level of capacity recuperation, more limited season of surgeries, less entanglements, higher AOFAS, EFAS, torment VAS scores, decreased plantar torment and decline reoperation rates. Most of the combination patients had great outcomes and bony association.
\end{abstract}

Keyword: Arthrodesis, Lisfranc, Ligamentous, Injury.

\section{Introduction}

Lisfranc wounds influence the tarsometatarsal (TMT), intercuneiform, and the naviculocuneiform joints. They incorporate any blend of hard and ligamentous injury to this complex [1].

Interosseous tendons join the bases of the second through fifth metatarsals. The Lisfranc tendon legitimate is a thick diagonal tendon stretching out from the base of the second metatarsal to the plantar part of the average cuneiform. The trustworthiness of this tendon is significant for solidness at the TMT verbalization, as there is no cross over metatarsal tendon between the first and second metatarsals just like the case between the lesser 4 metatarsals. On a cross-segment, the midfoot structures a cross over plantar curve made out of lopsidedly molded bones, with rigid and ligamentous life systems giving a "mortise" arrangement to the midfoot to help weight bearing powers. [2].

The Lisfranc tendon and its encompassing hard life structures in the midfoot involve a district of negligible anatomic development in the solid person. The absence of movement adds inflexibility to the average and cross over curves of the foot which balances out the design during exercises of day by day living. Wounds to the Lisfranc joint incorporate separations and breaks of the bones just as delicate tissue harm. These wounds can happen in high- energy mishaps like tumbles from a tallness or low- energy sports mishaps like inside revolution while the foot is plantar flexed. Lisfranc wounds bring about critical agony and uneasiness for the influenced individual, frequently forming into osteoarthritis and reformist weakness if not promptly treated. [3].
Most Lisfranc wounds are the aftereffect of a backhanded component of injury: $80 \%$ of patients endure roundabout wounds and $70 \%$ of those with Lisfranc crack disengagement support different wounds or qualify as polytraumatic. At the point when the foot is constrained into greatest plantar flexion, the more fragile dorsal tendons will tear and permit dorsal disengagement and break of the plantar part of the metatarsal bases. Extra powers will move the metatarsals on the bone structure, delivering kidnapping and sidelong dislodging, with pressure breaks of the tarsal bones, Chopart's joint, and the subtalar joint [4].

Lisfranc wounds can be bony, ligamentous, or a mix of the two. The first order framework by Quenu and Kuss depicted wounds as homolateral, detached, or dissimilar dependent on the bearing of the dislodged metatarsals. Hardcastle et al. further ordered Lisfranc wounds into Type $\mathrm{A}, \mathrm{B}$, or $\mathrm{C}$ dependent on relocation and incoherency with a framework that they thought would direct treatment. Myerson followed with changes to this framework dependent on course of separation regardless of these various arrangement plans, result and treatment don't dependably correspond with any injury type [5].

Finding of Lisfranc wounds is frequently difficult. Significant cracks and disengagements are generally clear with radiographs where the main discoveries are bone parts and plain diastasis (partition) between the base of the subsequent metatarsals and average cuneiform or between the average and delegate cuneiform. The test comes from wounds without net partitions or bone cracks. These are more normal in low- energy injury to the Lisfranc joint like those seen from sport wounds. In these cases, the delicate tissues 
and tendons of the midfoot are harmed and bring about joint shakiness and torment. Notwithstanding, 20-40\% Lisfranc wounds are missed in the underlying assessment since diastasis in the joint isn't handily observed. Up to half of those with Lisfranc wounds won't show an opening in the Lisfranc joint except if the radiographs are taken with weight- bearing. In any event, weight- bearing radiographs have been appeared to miss up to $15 \%$ of cases with Lisfranc wounds. [3].

Brief acknowledgment and treatment of Lisfranc injury is basic to limit the potential for huge long haul handicap. A high list of doubt is justified for these wounds since they are as often as possible unobtrusive or mysterious and can accordingly be barely noticeable. At the point when doubt is available in spite of the shortfall of identifi $\neg$ able anomaly on plain radiograph as $\neg$ sessment, weight-bearing radiographs and stress see assessments are recommend $\neg$ ed. General arrangement exists in the litera $\neg$ ture that anatomic decrease of the Lisfranc joint is significant for ideal result. [6].

Truly, open decrease and inner obsession was the acknowledged standard treatment for intense Lisfranc wounds. Combination was fundamentally utilized as a rescue methodology in circumstances where patients were at first treated with inward obsession and hence created post horrendous joint pain. Nonetheless, more as of late, essential combination has been proposed as authoritative administration for those patients who have broad articular ligament harm that makes post horrible joint inflammation unavoidable. [7].

Essential combination exhibits better results thought about than obsession while assessing unadulterated ligamentous or high energy Lisfranc wounds with extreme joint surface harm as it were. Different circumstances where essential combination has been suggested incorporate patients with postponed introductions and for stout or old patients in whom obsession has a higher pace of disappointment [7].

This examination intended to assess the transient consequences of essential arthrodesis in unadulterated ligamentous lisfranc wounds.

\section{Patients and methods}

This study has been conducted at the orthopedic department, faculty of medicine, Benha university hospitals on twenty patients, 13 males and 7 females with pure ligamentous lisfranc injuries.

\subsection{Inclusion criteria}

To be included in the study, the patient must fulfill all the following criteria:

- Pure ligamentous lisfranc injury.

- Skeletally mature patients.

- No sex limitations.

\subsection{Exclusion criteria}

The patients with any of the following criteria were excluded from the study:

- Inflammatory arthritis.

- Active infection.
- Severe vascular or neurological deficit affecting the lower limbs.

- Preoperative Management and Evaluation.

\subsection{Clinical evaluation}

A detailed sheet will be taken for all patients including:

- Personal history including age, sex, occupation, special habits of medical importance.

- History of present illness, side affected, previous treatment, past history and medical co- morbidities.

\subsection{Present history}

1)Type of fracture: Pure ligamentous lisfranc injury.

2) The mechanism of injury of included patients

3)Special habit: 4 patients were smokers $20 \%, 16$ patients were nonsmokers $80 \%$.

4)Medical history: 2 patients diabetic 10\%, 3 hepatitis c +ve $15 \%, 2$ patient's hypertensive $10 \%$ and 13 patient with no medical history $65 \%$.

\subsection{Past history}

1)no previous disease or injury to affected side.

2)Previous operative procedure 3 patients had appendectomy $15 \%$.

\subsection{Clinical examination \\ General Examination}

Pulse, blood pressure, pallor, level of consciousness..etc at the time of presentation all the patients were presented fully conscious.

\section{B. Local Examination}

Local examination of the affected side, vascular state, neurological state, skin condition, any signs of compartmental syndrome.

\section{Side of affection:}

The right side was affected in $12(60 \%)$ patients while the left side affected in $8(40 \%)$ patients Other associated injuries no patient

\subsection{Radiological evaluation} All patients will be examined radiologically by:

- Anteroposterior $\mathrm{x}$ ray of the foot.

- Oblique $\mathrm{x}$ ray of the foot.

- Lateral $x$ ray of the foot with stress dorsiflexion.

- C.T. scan.

\subsection{Operative intervention}

1)The procedure will be carry out under general or regional anesthesia.

2)Patient in supine position.

3) We prefer the dual-incision approach.

4)Primary arthrodesis of $1^{\text {st }}, 2$ nd, 3rd tarsometatarsal joints.

5)Back slab. 


\subsection{Operative Technique Positioning}

The patient is placed supine with a bolster beneath the ipsilateral hip. Protective padding is placed around the contralateral limb, primarily to protect the peroneal nerve, and the contralateral limb is secured to the table.

A sterile bolster is placed beneath the operative limb at the knee to facilitate access to the midfoot and intraoperative fluoroscopy.

\subsection{Approach}

We did single or dual-incision approach

\subsection{Post-operative Care}

In a primary arthrodesis, the limb is immobilized in serial short-leg non-weight-bearing cast for 10 to 12 weeks after surgery, at which point radiographic union is confirmed on weight-bearing radiographs.

The patient is converted to a venous compression stocking and prefabricated fracture boot and early progression to motion is initiated.

Weight bearing is not permitted unti110 to 12 weeks postoperatively, at which point weight-bearing radiographs are obtained to confirm maintenance of reduction.

The patient is gradually allowed to resume regular shoes, and activity is advanced as tolerated thereafter.

We do not routinely remove hardware unless symptomatic or specifically requested by the patient, in which case the implants may be removed at 1 year after surgery.

\section{Post-operative evaluation :}

All patients will be followed up for at least 12 months

1)AOFAS score comprises 3 areas pain function and alignment This is clinical administrated questionnaire scored out of 100

2)EFAS score European foot and ankle society score out of 40

3) Vas score of pain out of 10

4)X-ray at each follow-up, patients were assessed with

- Anteroposterior $\mathrm{x}$ ray of the foot.

- Oblique x ray of the foot.

- Lateral $x$ ray of the foot with stress dorsiflexion.

\section{Results}

The present study was done on 20 patients with acute ligamentous lisfranc injury who were treated operatively using primary arthrodesis.

The mean age of the included patients was $27.4 \pm$ 6.19 years old with minimum age 19 years old and maximum age 39 years old. 4 patients were smokers $20 \%, 16$ patients were nonsmoker's $80 \% .2$ patients diabetic $10 \%, 3$ hepatitis $\mathrm{c}+\mathrm{ve} 15 \%, 2$ patients hypertensive $10 \%$ and 13 patient with no medical history $65 \%$.

Table (1) Demographic characteristics in study population

\begin{tabular}{|c|c|c|}
\hline \multicolumn{3}{|c|}{ General characteristics } \\
\hline Age (years) & Mean \pm SD & $27.4 \pm 6.19$ \\
\hline Gender & $\begin{array}{ll}\text { Male } & \mathrm{n}(\%) \\
\text { Female } & \mathrm{n}(\%)\end{array}$ & $\begin{array}{l}13(65 \%) \\
7(35 \%)\end{array}$ \\
\hline
\end{tabular}

The mechanism of injury of the included patients. The most common mechanism of injury was road traffic accident $(55 \%)$, followed by fall from height (40\%), and followed by hyper plantar flexion foot trauma during descending stairs $(5 \%)$.

Table 2 Distribution of the studied group according to mechanism of injury

\begin{tabular}{lcc}
\hline MECHANISM of injury & No & Percent \\
\hline RTA & 11 & $55 \%$ \\
Fall from height & 8 & $40 \%$ \\
Hyper plantar flexion foot trauma during descending stairs & 1 & $5 \%$ \\
\hline
\end{tabular}

Mean AOFAS score differences according to sex shows no statistical significance between mean AOFAS regarding sex. ( $\mathrm{P}$ value was 0.31 ) ( $\mathrm{P}$ value was $>0.05$ ). Mean AOFAS score differences according to smoking shows no statistical significance between mean AOFAS regarding smoking. ( $\mathrm{P}$ value was 0.13 ) ( $\mathrm{P}$ value was $>$ 0.05). Mean AOFAS score differences according to medical history shows no statistical significance between mean AOFAS regarding medical history. ( $\mathrm{P}$ value was 0.49 ) ( $\mathrm{P}$ value was $>0.05$ ). Mean AOFAS score differences according to mechanism of injury shows no statistical significance between mean AOFAS regarding mechanism of injury. ( $\mathrm{P}$ value was 0.24$)(\mathrm{P}$ value was $>0.05)$. 


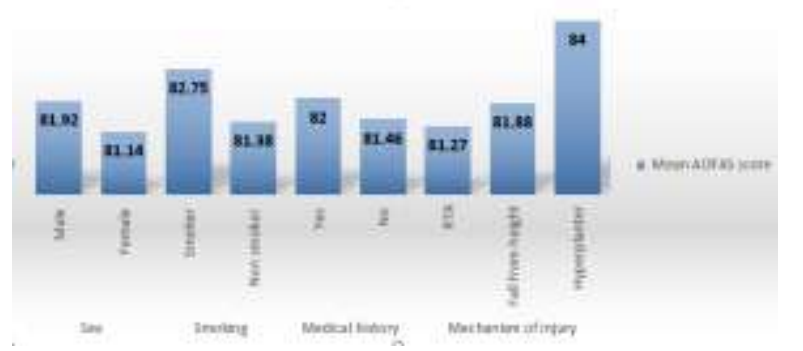

Fig. (1) Mean AOFAS score differences according to sex, smoking, medical history and mechanism of injury.

Mean EFAS score differences according to sex shows no statistical significance between mean EFAS regarding sex. ( $\mathrm{P}$ value was 0.052 ) ( $\mathrm{P}$ value was $>$ 0.05). Mean EFAS score differences according to smoking shows no statistical significance between mean EFAS regarding smoking. ( $\mathrm{P}$ value was 0.26) ( $\mathrm{P}$ value was $>0.05)$. Mean EFAS score differences according to medical history shows no statistical significance between mean EFAS regarding medical history. ( $\mathrm{P}$ value was 0.64 ) ( $\mathrm{P}$ value was $>0.05$ ). Mean EFAS score differences according to mechanism of injury shows no statistical significance between mean EFAS regarding mechanism of injury. ( $\mathrm{P}$ value was 0.30) (P value was $>0.05)$.

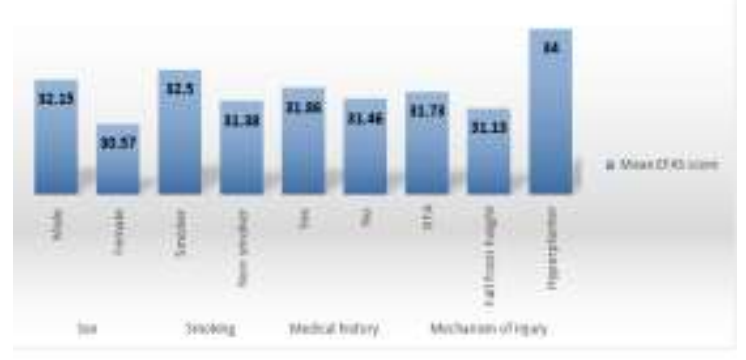

Fig. (2) Mean EFAS score differences according to sex, smoking, medical history and mechanism of injury.

Mean pain VAS score differences according to sex shows no statistical significance between mean pain VAS score regarding sex. ( $\mathrm{P}$ value was 0.70 ) ( $\mathrm{P}$ value was $>0.05)$. Mean pain VAS score differences according to smoking shows no statistical significance between mean pain VAS score regarding smoking. ( $\mathrm{P}$ value was 0.39$)(\mathrm{P}$ value was $>0.05)$. Mean pain VAS score differences according to medical history shows no statistical significance between mean pain VAS score regarding medical history. ( $\mathrm{P}$ value was 0.15 ) ( $\mathrm{P}$ value was $>0.05)$. Mean pain VAS score differences according to mechanism of injury shows no statistical significance between mean pain VAS score regarding mechanism of injury. ( $\mathrm{P}$ value was 0.38 ) ( $\mathrm{P}$ value was $>$ $0.05)$.

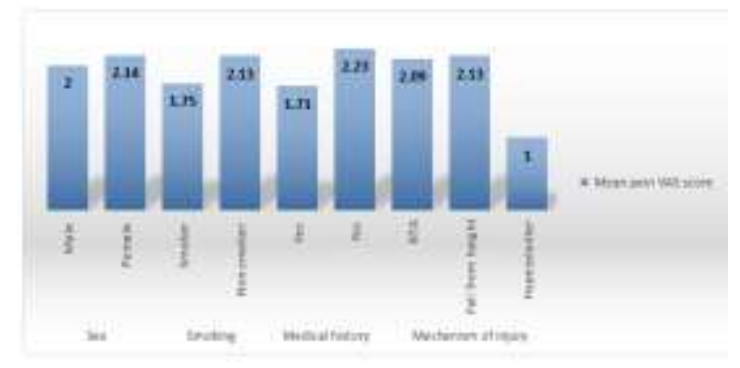

Fig. (3) Mean pain VAS score differences according to sex, smoking, medical history and mechanism of injury.

Mean union time differences according to sex shows no statistical significance between mean union time regarding sex. ( $\mathrm{P}$ value was 0.90$)$ ( $\mathrm{P}$ value was $>$ 0.05). Mean union time differences according to smoking shows no statistical significance between mean union time regarding smoking. ( $\mathrm{P}$ value was 0.83 ) $(\mathrm{P}$ value was $>0.05)$. Mean union time differences according to medical history shows no statistical significance between mean union times regarding medical history. ( $\mathrm{P}$ value was 0.30 ) ( $\mathrm{P}$ value was $>$ 0.05) Mean union time differences according to mechanism of injury shows no statistical significance between mean union times regarding mechanism of injury. ( $\mathrm{P}$ value was 0.26 ) ( $\mathrm{P}$ value was $>0.05$. 


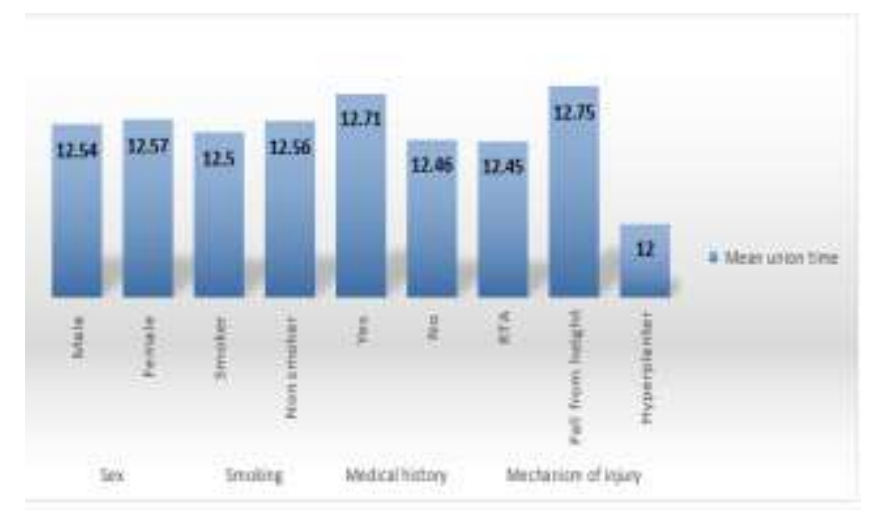

Fig. (4) Mean union time differences according to sex, smoking, medical history and mechanism of injury.

Correlation between AOFAS score, EFAS score, pain VAS score and union time shows no statistical significance regarding correlation between AOFAS score (P value was 0.139) (Figure 1), EFAS score (P value was 0.542) (Figure 2), pain VAS score (P value was 0.152) (Figure 3) and union time (P value was $>$ 0.05 ) (Figure 4).

Table (3) Correlation between AOFAS score, EFAS score, pain VAS score and union time.

\begin{tabular}{llc}
\hline & & Union time \\
\hline AOFAS score & Pearson Correlation & -0.34 \\
& P value & 0.139 \\
EFAS score & Pearson Correlation & -0.145 \\
\multirow{2}{*}{ Pain VAS score } & P value & 0.542 \\
& Pearson Correlation & 0.33 \\
& P value & 0.152 \\
\hline
\end{tabular}

\section{Discussion}

The current examination was a clinical report that was led on a sequential 20 patients with unadulterated ligamentous lisfranc injury who were dealt with operatively utilizing essential arthrodesis at Benha college emergency clinics.

In the current examination, the mean age of the included patients was $27.4 \pm 6.19$ years old and most of them were guys $(65 \%)$.

The most widely recognized component of injury was street car crash (55\%), trailed by tumble from stature (40\%), and followed by hyper plantar flexion foot injury during plummeting steps $(5 \%)$.

In the current examination, the mean AOFAS score of the included patients was $81.65 \pm 1.60$ with least score 80 and most extreme score 84 .

In concordance with our discoveries, SHEIBANIRAD, Shahin, et al. uncovered that the mean AOFAS score was higher in the essential arthrodesis bunch at 1year follow-up for unadulterated ligamentous and consolidated hard and ligamentous wounds. Additionally support essential arthrodesis as an essential treatment for Lisfranc joint wounds because of an altogether diminished pace of ad $\neg$ ditional medical procedures, just as an inclination toward improved clinical result scores when contrasted and ORIF [6].

Likewise, QIAO, Yusen, et al. uncovered that Patients in the arthrodesis bunch had a higher AOFAS score contrasted and patients in the non-combination bunch [8].
In the current investigation, the mean EFAS score of the included patients was $31.60 \pm 1.76$ with least score 28 and greatest score 34 .

In the current investigation, the mean Pain VAS score of the included patients was $2.05 \pm 0.76$ with least score 1 and greatest score 3 .

In concordance with our discoveries, ETTINGER, Sarah, et al. uncovered that TMT arthrodesis of the average and focal segment brought about critical improvement in foot capacity and agony [9].

Likewise, YAN, Alan, et al. uncovered that for high-energy Lisfranc wounds with clear break disengagement and complete ligamentous disturbance, essential arthrodesis of the average and center sections is liked [7].

In the current investigation, the mean Union season of the included patients was $12.55 \pm 0.51$ weeks with least 12 weeks and most extreme 13 weeks.

In the current investigation, no measurable importance was found between mean AOFAS in regards to sex (P esteem was 0.31), smoking (P esteem was 0.13$)$, clinical history ( $\mathrm{P}$ esteem was 0.49 ) and system of injury ( $\mathrm{P}$ esteem was 0.24 ) ( $\mathrm{P}$ esteem was $>$ $0.05)$.

In the current examination, no measurable importance was found between mean EFAS in regards to sex. ( $\mathrm{P}$ esteem was 0.052), smoking ( $\mathrm{P}$ esteem was $0.26)$, clinical history ( $\mathrm{P}$ esteem was 0.64$)$ and instrument of injury (P esteem was 0.30 ) (P esteem was $>0.05)$. 
In the current examination, no factual importance was found between mean agony VAS score with respect to sex ( $\mathrm{P}$ esteem was 0.70$)$, smoking ( $\mathrm{P}$ esteem was 0.39), clinical history ( $\mathrm{P}$ esteem was 0.15$)$ and component of injury. ( $\mathrm{P}$ esteem was 0.38) ( $\mathrm{P}$ esteem was $>0.05)$.

In the current examination, shows no factual importance was found between mean association times with respect to sex ( $\mathrm{P}$ esteem was 0.90), smoking ( $\mathrm{P}$ esteem was 0.83 ), clinical history ( $\mathrm{P}$ esteem was 0.30 ) and system of injury (P esteem was 0.26 ) (P esteem was $>0.05)$.

In the current investigation, shows no factual importance with respect to relationship between's AOFAS score ( $\mathrm{P}$ esteem was 0.139), EFAS score ( $\mathrm{P}$ esteem was 0.542$)$ and torment VAS score ( $\mathrm{P}$ esteem was 0.152 ) and association time. (P esteem was $>0.05$ ).

\section{Conclusion}

Lisfranc wounds are mind boggling and care should be taken in choosing the suitable treatment. Essential arthrodesis in unadulterated ligamentous lisfranc injury has benefits: diminished foot distortion rates, supported biomechanical morphology of the feet, decreased difficulties, more significant level of capacity recuperation, more limited season of surgeries, less complexities, higher AOFAS, EFAS, torment VAS scores, diminished plantar agony and decline reoperation rates. Most of the combination patients had great outcomes and rigid association.

As per our exploration, essential arthrodesis might be a superior decision for treating unadulterated ligamentous Lisfranc injury.

\section{References}

[1] N.Smith , C.Stone , A.Furey . Does open reduction and internal fixation versus primary arthrodesis improve patient outcomes for Lisfranc trauma? A systematic review and meta-analysis. Clinical Orthopaedics and Related Research®. Jun.vol. 1;474(6),pp.1445-52,2016.

[2] J. Lewis , R. Anderson . Lisfranc injuries in the athlete. Foot \& Ankle International. Dec.vol.37(12),pp.1374-80,2016.

[3] J.OWEN. Computational Analysis of the Clinical Presentation of a Ligamentous Lisfranc Injury. Journal of Orthopaedic Research: Official Publication of the Orthopaedic Research Society.vol.8,pp.22-33,2021.

[4] J.van Rijn , D.Dorleijn , B.Boetes, WiersmaTuinstra, S.Moonen. Missing the Lisfranc fracture: a case report and review of the literature. The Journal of foot and ankle surgery. Ma.vol.r 1;51(2),pp.270-4,2012.

[5] T.Schepers , P.Oprel , E.Van Lieshout . Influence of approach and implant on reduction accuracy and stability in Lisfranc fracturedislocation at the tarsometatarsal joint. Foot \& ankle international. May.vol.34(5),pp.705-10,2013.

[6] RSHEIBANI And Shahin . Arthrodesis versus ORIF for Lisfranc fractures. Orthopedics.vol.35.6,pp. e868-e873,2012.

[7] YAN And Alan . Updates on Lisfranc Complex Injuries. Foot \& Ankle Orthopaedics.vol. 6.1,pp. 2473011420982275, 2021.

[8] QIAO And Yu- sen . Comparison of Arthrodesis and Non- fusion to Treat Lisfranc Injuries. Orthopaedic surgery.vol. 9.1,pp. 62$68,2017$.

[9] ETTINGER And Sarah. Comparison of Isolated Screw to Plate and Screw Fixation for Tarsometatarsal Arthrodesis Including Clinical Outcome Predictors. Foot \& Ankle International.vol.8,pp.1071100720980014,2021. 